\title{
História gestacional e características da assistência pré-natal de puérperas adolescentes e adultas em uma maternidade do interior de Minas Gerais, Brasil
}

\author{
Gestational history and prenatal care characteristics of adolescent \\ and adult mothers in a maternity hospital in the interior of Minas \\ Gerais, Brazil
}

Luciana Angélica Vieira Santos ${ }^{1}$

Maristela Oliveira Lara ${ }^{1}$

Renata Caroline Ribeiro Lima ${ }^{1}$

André Freire Rocha ${ }^{1}$

Euza Mara Rocha ${ }^{2}$

José Cristiano Ramos Glória ${ }^{3}$

Gabriela de Cássia Ribeiro ${ }^{1}$

${ }^{1}$ Departamento de Enfermagem, Universidade Federal dos Vales do Jequitinhonha e Mucuri (UFVJM). Rodovia MGT $367 / \mathrm{Km} 583 / 5000$, Alto da Jacuba. 39100-000 Diamantina MG Brasil. luangelica5@hotmail.com

${ }^{2}$ Fundação Hemominas.

Diamantina MG Brasil

${ }^{3}$ Departamento de Odontologia, UFVJM.

Diamantina MG Brasil.
Abstract The scope of this research was to analyze the gestational history and prenatal care characteristics of adolescent and adult mothers in a maternity hospital located in a city in Minas Gerais, which is a hospital of reference in the macro-region of health of Jequitinhonha. It involved a descriptive cross-sectional study. A total of 327 mothers were interviewed between May 2013 and March 2014 using a semi-structured questionnaire. With a sample of 255, the number of adult women was predominant. With respect to prenatal care, 324 pregnant women had medical appointments. In terms of the location for prenatal care, $79.2 \%$ of adolescents were attended in the public health service, while that percentage was $60.4 \%$ among adult women. Regarding the type of birth, $54.7 \%$ of mothers had normal delivery and 45\% had cesarean section. Among adolescents, there was a higher percentage of normal delivery compared to adult women and this data had a statistically significant relationship with the age of the pregnant women. With respect to gestational age at birth, $85.9 \%$ had full-term deliveries, $13.5 \%$ had preterm delivery and $0.6 \%$ had post-term delivery. It was revealed that adolescent mothers were at a disadvantage compared to the other mothers in terms of both socioeconomic characteristics and prenatal care received.

Key words Prenatal care, Adolescent pregnancy, Pregnancy
Resumo O objetivo desta pesquisa foi analisar a história gestacional e as características da assistência pré-natal de puérperas adolescentes e adultas em uma maternidade localizada em uma cidade de Minas Gerais, referência para a macrorregião de saúde do Jequitinhonha. Trata-se de um estudo transversal descritivo. Entrevistou-se 327 puérperas, entre maio de 2013 a março de 2014, utilizando um instrumento semiestruturado. Predominou o número de puérperas adultas com uma amostra de 255. Com relação ao pré-natal, 324 puérperas realizaram as consultas. Quanto ao local de realização do pré-natal, 79,2\% das adolescentes, realizaram no serviço público de saúde, enquanto entre as adultas essa porcentagem foi de 60,4\%. Quanto ao tipo de parto, 54,7\% das puérperas o tiveram normal e $45 \%$ cesárea. Entre as adolescentes, houve uma maior porcentagem de parto normal comparado às adultas, e esse dado teve relação estatisticamente significativa com a idade da puérpera. Com relação à idade gestacional no momento do parto, 85,9\% tiveram seus partos a termo; $13,5 \%$ pré-termo e 0,6\% pós-termo. Evidenciou-se que as puérperas adolescentes estiveram em desvantagem em relação às demais mães no que diz respeito tanto às características socioeconômicas quanto na assistência recebida no pré-natal.

Palavras-chave Cuidado pré-natal, Gravidez na adolescência, Gravidez 


\section{Introdução}

A cada cinco mulheres brasileiras, uma tem o primeiro filho na adolescência, ou seja, antes dos 20 anos de idade, e esta proporção tem se mantido constante nos últimos 10 anos $^{1}$. A maternidade na adolescência constitui uma ocorrência de repercussão mundial, cuja acepção diverge nas diferentes culturas e contextos, representando um desafio para as políticas públicas, especialmente no domínio da saúde, uma vez que pode acarretar problemas psicossociais, econômicos e complicações obstétricas que comprometem a saúde materna e do neonato. A literatura mundial tem evidenciado que a importância da maternidade na adolescência transcende os aspectos clínicos, uma vez que fatores sociais, econômicos e culturais interagem, causando impactos que podem ser positivos ou negativos no estado de saúde materno e fetal ${ }^{2,3}$.

As taxas de fecundidade adolescente da América Latina são superiores às médias internacionais, que variam entre $3,4 \%$ a $7,6 \%$ em países desenvolvidos, entretanto continua sendo muito alta em termos de comparação, apresentando incidência maior nas camadas socioeconômicas mais desfavorecidas da população².

O acompanhamento ao pré-natal é o período anterior ao nascimento da criança e constitui-se um conjunto de procedimentos clínicos e educativos com o objetivo de vigiar a evolução da gravidez, bem como, orientar e esclarecer a mulher e sua família sobre a gestação, o parto e os cuidados com o recém-nascido. Busca ainda prevenir, detectar precocemente e tratar as intercorrências mais frequentes nesse período ${ }^{4}$. Observou-se no Brasil, no ano de 2010, o aumento da cobertura da assistência pré-natal e do número de consultas por gestantes nos últimos 15 anos, sendo a proporção de gestantes sem acesso a qualquer consulta de pré-natal inferior a $2 \% \%^{5,6}$.

Pesquisas tem demonstrado que a gravidez na adolescência pode estar relacionada à maior ocorrência de prematuridade, baixo peso ao nascer (o que pode predispor o recém-nascido a infecções ou a problemas como hipoglicemia, hipóxia e atraso no desenvolvimento neuropsicomotor futuro), pré-natal inadequado, que implica menor número de consultas, início das consultas mais tardiamente e baixa realização de exames complementares. Pode somar-se a estes fatores a falta de informações sobre os benefícios do pré-natal e o sentimento de vergonha pela busca de cuidados médicos por parte das adolescentes ${ }^{1,7}$.
Diante desse fato, a atenção pré-natal de qualidade e humanizada é fundamental para a saúde, tanto das mães adolescentes quanto adultas e do neonato, por incluir ações de prevenção e promoção da saúde da gestante e do feto, identificando precocemente possíveis situações de risco para ambos e possibilitando intervenções adequadas. Apesar da cobertura do pré-natal estar aumentando no Brasil, estudo sobre a qualidade do atendimento mostra que ainda há muito que avançar8. Para os profissionais de saúde, a relevância deste trabalho consiste no conhecimento dos dados levantados pela pesquisa, assim como das propostas dela resultantes, servindo de instrumento de reflexão para a adequação e melhoria da assistência prestada.

O objetivo desse estudo foi analisar a história gestacional e características da assistência pré-natal de puérperas adolescentes e adultas em uma maternidade localizada em uma cidade do interior de Minas Gerais, referência para a macrorregião de saúde do Jequitinhonha.

\section{Metodologia}

Trata-se de um estudo transversal descritivo. Essa investigação faz parte do escopo de uma pesquisa maior intitulada "Estudo do perfil epidemiológico da gravidez entre puérperas adolescentes e adultas", cujo objetivo principal foi analisar o perfil da gravidez segundo aspectos socioeconômicos, demográficos, de gestação e tipo de parto. O público-alvo foram puérperas adolescentes (sendo consideradas adolescentes, segundo a Organização Mundial da Saúde - OMS 9 , mulheres com idade menor ou igual a 20 anos) e adultas, mulheres com idade superior a 20 anos, que tiveram seus partos na maternidade localizada no Hospital Nossa Senhora da Saúde, no município de Diamantina-MG, no período de maio de 2013 a março de 2014.

A seleção da amostra, inicialmente, realizouse pelo levantamento no banco de dados oficial, DATASUS, sobre o número de partos ocorridos no município de Diamantina, Minas Gerais. O último período informado foi o ano de 2010, contabilizando 1295 partos $^{10}$. Foi realizado, em um segundo momento, uma amostragem aleatória simples considerando o intervalo de confiança de $95 \%$ e ponderando as possíveis perdas, chegando ao total de 327 puérperas.

Os critérios de inclusão foram aceitar participar da pesquisa (independente da faixa etária), ter o parto realizado na maternidade do municí- 
pio de Diamantina-MG, que é referência para a macrorregião do Jequitinhonha, e estar em período de internação na referida maternidade.

Para a coleta de dados foi utilizado um instrumento semiestruturado contendo questões relativas aos aspectos socioeconômicos, demográficos, de gestação e parto, história reprodutiva, comportamento sexual e contraceptivo, suporte social e afetivo e hábitos de vida.

Foram realizados anteriormente oito testespiloto para verificar a adequação do instrumento de coleta de dados, não foi necessário realizar alterações significativas no mesmo. Duas puérperas por dia foram entrevistadas, considerando o período de segunda a sexta-feira, sendo selecionadas as mulheres que tiveram os dois primeiros partos do dia. Cada uma delas foi convidada a assinar um Termo de Consentimento Livre e Esclarecido (TCLE). Após a entrevista realizavase a investigação de informações necessárias no prontuário e no cartão de pré-natal de forma a complementar as informações buscadas.

Os dados extraídos foram armazenados no banco de dados do Programa Statistical Package for the Social Sciences (SPSS) versão 20.0. Posteriormente, após verificação de possíveis incoerências, os resultados foram analisados utilizando-se a estatística simples, com frequências absolutas e relativas, Teste Qui-quadrado e Teste Exato de Fisher, considerando correlações estatisticamente significativas às que obtiveram $p<$ 0,05 e foram discutidos com base na literatura.

Esta pesquisa foi apreciada e aprovada pelo Comitê de Ética e Pesquisa da Universidade Federal dos Vales do Jequitinhonha e do Mucuri CEP/UFVJM.

\section{Resultados}

Neste estudo, foi predominante o número de puérperas adultas com uma amostra de 255 $(78,0 \%)$ em relação a $72(22,0 \%)$ adolescentes. A média de idade foi de 26,7 com um desvio padrão de 6,8, sendo os extremos de 15 e 45 anos de idade. A Tabela 1 descreve características demográficas e socioeconômicas das participantes. Os resultados mostram que as mães adultas são em sua maioria casadas ou estão em uma união estável $(82,4 \%)$, diferente do grupo de mães adolescentes, em que apenas pouco mais da metade vive com o companheiro. Em relação ao tempo de casado, observa-se que a maioria das mulheres que convivem com seus parceiros há mais de um ano, são também as adultas. Houve relação estatística com relação ao estado civil e tempo de casado.

Relacionado à escolaridade, aproximadamente dois terços das puérperas possuem ensino médio ou superior, não tendo esse dado relação com a idade materna. Já em relação a com quem morava quando engravidou, as adolescentes residiam, em sua maioria $(58,3 \%)$, com os familiares, enquanto as puérperas adultas moravam com o marido $(67,5 \%)$.

Quanto à situação de trabalho, a grande maioria das adolescentes $(95,8 \%)$ não trabalha, enquanto entre as adultas um pouco mais da metade não trabalha, atualmente. Tendo em consideração a renda mensal das puérperas, $35,7 \%$ das adultas recebem entre um e três salários mínimos, enquanto $5,6 \%$ das adolescentes recebem essa mesma faixa de valor.

No que se refere aos riscos gestacionais, $18,1 \%$ das puérperas adolescentes e 17, $4 \%$ das adultas afirmaram terem feito uso de bebida alcóolica durante a gravidez. Com relação ao cigarro, 5,6\% das adolescentes e 7,8\% das adultas fumaram durante a gravidez. Quanto aos problemas de saúde durante a gestação, $51,4 \%$ das adolescentes e $53,7 \%$ das adultas tiveram algum tipo de intercorrência. Os problemas mais ocorrentes foram anemia (35\%), Infecção do Trato Urinário (ITU) (34,3\%), Doença Hipertensiva Específica da Gravidez (DHEG) (18\%) e Pré-eclâmpsia (14\%). Esses dados não se relacionam estatisticamente com a idade da puérpera, exceto a DHEG, que foi prevalente somente entre as adultas (Tabela 2).

A Tabela 3 descreve a assistência à gestação. Tendo em consideração o pré-natal, $99,1 \%$ de todas as puérperas realizaram as consultas, sendo que três não tiveram este acompanhamento alegando desconhecimento da gestação. Quanto ao local de realização do pré-natal, $79,2 \%$ das adolescentes realizaram no serviço público de saúde; $4,2 \%$, em consultório particular ou convênio, e 13,9\%, em mais de um local (como, por exemplo, Estratégia de Saúde da Família e particular, ou Estratégia de Saúde da Família e unidade de atenção secundária). Esse dado foi estatisticamente significativo. Entre as adultas, $60,4 \%$ realizaram no serviço público; $11,8 \%$, particular ou convênio, e $26,3 \%$, em mais de um local.

Relacionado à internação, $12,5 \%$ das puérperas adolescentes e $16,1 \%$ das adultas ficaram internadas por pelo menos 24 horas durante a gravidez (Tabela 3). As principais causas de internação foram hipertensão $(3,4 \%)$, infecção urinária $(2,4 \%)$; hemorragia $(0,9 \%)$ e Pré-eclâmpsia $(0,9 \%)$. Esse dado não apresentou significância estatística com a idade. 
Tabela 1. Características sociodemográficas e econômicas das puérperas que tiveram seus partos em uma maternidade de referência para a macrorregião de saúde de Diamantina - MG, maio de 2013 a março de 2014.

\begin{tabular}{|c|c|c|c|}
\hline $\begin{array}{c}\text { Características sociodemográficas e } \\
\text { econômicas }\end{array}$ & $\begin{array}{c}\text { Adolescentes } \\
\text { n (\%) }\end{array}$ & $\begin{array}{c}\text { Adultas } \\
\text { n (\%) }\end{array}$ & Valor de $\mathrm{p}$ \\
\hline \multicolumn{4}{|l|}{ Estado civil } \\
\hline Casado/união estável & $40(55,6)$ & $210(82,4)$ & \\
\hline Solteiro/separado/divorciado & $32(44,40)$ & $45(17,6)$ & $<0,001^{\star}$ \\
\hline \multicolumn{4}{|l|}{ Tempo de casado } \\
\hline Até um ano & $18(45,0)$ & $37(17,6)$ & \\
\hline Mais que 1 ano & $22(55,0)$ & $173(82,4)$ & $<0,001^{\star}$ \\
\hline \multicolumn{4}{|l|}{ Escolaridade } \\
\hline Até ensino fundamental & $24(33,3)$ & $87(34,1)$ & \\
\hline Ensino médio ou superior & $48(66,7)$ & $168(65,9)$ & $0,901^{\star}$ \\
\hline \multicolumn{4}{|l|}{ Com quem morava quando engravidou } \\
\hline Marido/companheiro & $19(26,4)$ & $172(67,5)$ & \\
\hline Familiares & $42(58,3)$ & $54(21,2)$ & \\
\hline Amigo/sozinha & $1(1,4)$ & $8(3,1)$ & \\
\hline Marido/familiares & $10(13,9)$ & $20(7,8)$ & \\
\hline Outros & $0(0,0)$ & $1(0,4)$ & $<0,001^{\star *}$ \\
\hline \multicolumn{4}{|l|}{ Trabalha atualmente } \\
\hline Sim & $3(4,2)$ & $119(46,7)$ & \\
\hline Não & $69(95,8)$ & $136(53,3)$ & $<0,001^{\star *}$ \\
\hline \multicolumn{4}{|l|}{ Renda mensal } \\
\hline Menos de 1 salário mínimo & $3(4,2)$ & $29(11,4)$ & \\
\hline 1 a 3 salários mínimos & $4(5,6)$ & $91(35,7)$ & \\
\hline Mais de 3 salários mínimos & $0(0,0)$ & $14(5,5)$ & \\
\hline Não se aplica & $65(72,0)$ & $121(47,5)$ & $<0,001^{\star}$ \\
\hline
\end{tabular}

Tabela 2. Riscos gestacionais das puérperas que tiveram seus partos em uma maternidade de referência para a macrorregião de saúde de Diamantina - MG, maio de 2013 a março de 2014.

\begin{tabular}{|c|c|c|c|}
\hline Riscos gestacionais & $\begin{array}{c}\text { Adolescentes } \\
\text { n (\%) }\end{array}$ & $\begin{array}{c}\text { Adultas } \\
\text { n (\%) }\end{array}$ & Valor de $\mathrm{p}$ \\
\hline \multicolumn{4}{|l|}{ Usou bebida alcoólica durante a gravidez } \\
\hline Sim & $13(18,1)$ & $44(17,3)$ & \\
\hline Não & $59(81,9)$ & $211(82,7)$ & $0,874^{\star}$ \\
\hline \multicolumn{4}{|l|}{ Fumou durante a gravidez } \\
\hline Sim & $4(5,6)$ & $20(7,8)$ & \\
\hline Não & $68(94,4)$ & $235(92,2)$ & $0,511^{\star}$ \\
\hline \multicolumn{4}{|l|}{ Problema de saúde durante a gravidez } \\
\hline Não & $35(48,6)$ & $118(46,3)$ & \\
\hline Sim & $37(51,4)$ & $137(53,7)$ & $0,726^{*}$ \\
\hline Infecção urinária & $23(31,9)$ & $89(34,9)$ & $0,641^{\star}$ \\
\hline Anemia & $12(16,7)$ & $23(9,0)$ & $0,064^{*}$ \\
\hline Doença Hipertensiva Específica da Gravidez & $0(0)$ & $18(7,1)$ & $0,017^{\star \star}$ \\
\hline Pré-eclâmpsia & $2(2,8)$ & $12(4,7)$ & $0,74^{\star *}$ \\
\hline
\end{tabular}

*Teste Qui-quadrado; ${ }^{* *}$ Teste Exato de Fisher.

Em referência ao início do pré-natal, a média de idade gestacional para realizar a primeira consulta de pré-natal das adolescentes foi de 2,9 meses com desvio padrão de 1,4 , sendo que a média das puérperas adultas foi de 2,2 meses com desvio padrão de 1,2, este dado teve importância 
significativa, ou seja, as adolescentes iniciaram o pré-natal mais tardiamente que as adultas.

Quanto às consultas de pré-natal, a maioria das puérperas $(84,7 \%)$ realizou o número adequado de consultas (maior ou igual a 6), entretanto, essa porcentagem foi maior entre as adultas. A média de consultas das puérperas adolescentes, conforme a tabela 3 , foi de 7,3 com um desvio padrão de 2,5 , enquanto das adultas foi de 8,4 , com os extremos de 0 e 18 consultas.

A maior parte dos partos foi coberta pelo Sistema Único de Saúde - SUS (91,7\%), enquanto $8,3 \%$ das mulheres tiveram seus partos por con- vênio ou particular. Entre as adolescentes, somente um parto foi particular, enquanto entre as adultas, $89,8 \%$ dos partos foram realizados pelo SUS e $10,2 \%$ foram por meio de convênio ou particular.

A Tabela 4 apresenta a história gestacional. Quanto ao tipo de parto, 54,7\% das puérperas tiveram parto normal e $45 \%$ tiveram parto cesárea. Entre as adolescentes, houve uma maior porcentagem de parto normal comparado às adultas. Com relação a idade gestacional no momento do parto, $85,9 \%$ tiveram seus partos a termo; $13,5 \%$ tiveram parto pré-termo e $0,6 \%$ tiveram parto

Tabela 3. Assistência à gestação das puérperas que tiveram seus partos em uma maternidade de referência para a macrorregião de saúde de Diamantina - MG, maio de 2013 a março de 2014.

\begin{tabular}{|c|c|c|c|}
\hline Assistência à gestação & $\begin{array}{c}\text { Adolescentes } \\
\text { n (\%) }\end{array}$ & $\begin{array}{c}\text { Adultas } \\
\text { n (\%) }\end{array}$ & Valor de $\mathrm{p}$ \\
\hline \multicolumn{4}{|l|}{ Pré-natal durante a gravidez } \\
\hline $\operatorname{Sim}$ & $71(98,6)$ & $253(99,2)$ & \\
\hline Não & $1(1,4)$ & $2(0,8)$ & $0,635^{* *}$ \\
\hline \multicolumn{4}{|l|}{ Local de realização do pré-natal } \\
\hline Estratégia de Saúde da Família & $57(79,2)$ & $153(60,4)$ & \\
\hline Particular/Convênio & $3(4,2)$ & $30(11,8)$ & \\
\hline Unidade de atenção secundária & $1(1,4)$ & $2(0,8)$ & \\
\hline Mais de um local & $10(13,9)$ & $67(26,3)$ & $0,034^{\star *}$ \\
\hline \multicolumn{4}{|l|}{ Internação durante a gravidez } \\
\hline Sim & $9(12,5)$ & $41(16,1)$ & \\
\hline \multirow[t]{2}{*}{ Não } & $63(87,5)$ & $214(83,9)$ & $0,456^{*}$ \\
\hline & Média (DP) & Média (DP) & \\
\hline Meses de gravidez quando iniciou pré-natal & $2,9(1,4)$ & $2,2(1,2)$ & $<0,001^{\star}$ \\
\hline Número de consultas & $7,3(2,5)$ & $8,4(2,5)$ & $<0,001^{*}$ \\
\hline
\end{tabular}

${ }^{\star}$ Teste Qui-quadrado; ${ }^{\star *}$ Teste Exato de Fisher.

Tabela 4. História gestacional das puérperas que tiveram seus partos em uma maternidade de referência para a macrorregião de saúde de Diamantina - MG, maio de 2013 a março de 2014.

\begin{tabular}{|c|c|c|c|}
\hline História gestacional & $\begin{array}{l}\text { Adolescentes } \\
\mathbf{n}(\%)\end{array}$ & $\begin{array}{c}\text { Adultas } \\
\text { n (\%) }\end{array}$ & Valor de $\mathrm{p}$ \\
\hline \multicolumn{4}{|l|}{ Tipo de parto } \\
\hline Normal/fórceps & $51(70,8)$ & $129(50,6)$ & \\
\hline Cesárea & $21(29,2)$ & $126(49,4)$ & $0,002^{*}$ \\
\hline \multicolumn{4}{|c|}{ Idade gestacional no momento do parto } \\
\hline Menor que 37 semanas & $9(12,5)$ & $35(13,7)$ & \\
\hline Entre 37 e 42 semanas & $62(86,1)$ & $219(85,9)$ & \\
\hline Maior que 42 semanas & $1(1,4)$ & $1(0,4)$ & $0,637^{\star *}$ \\
\hline \multicolumn{4}{|l|}{ Peso do recém-nascido } \\
\hline Baixo peso & $2(2,8)$ & $2(0,8)$ & \\
\hline Peso insuficiente & $6(8,3)$ & $31(12,2)$ & \\
\hline Peso adequado & $63(87,5)$ & $210(82,4)$ & \\
\hline Excesso de peso & $1(1,4)$ & $12(4,7)$ & $0,477^{\star *}$ \\
\hline
\end{tabular}

* Teste Qui-quadrado; ** Teste Exato de Fisher. 
pós-termo. No que diz respeito ao peso do recém-nascido, 83,5\% dos recém-nascidos nasceram com peso adequado; $11,3 \%$, nasceram com peso insuficiente e $4 \%$, nasceram com excesso de peso. A idade gestacional no momento do parto e o peso do recém-nascido não tiveram associação estatística com a idade materna.

A principal intercorrência ocorrida no parto foi a desproporção céfalo-pélvica (3,4\%), seguida da eclampsia $(2,8 \%)$ e apresentação anômala do feto $(2,4 \%)$.

\section{Discussão}

No presente estudo, a porcentagem das mães adolescentes é semelhante a observada para o Brasil em $2010(21,2 \%)$ e cerca de 5\% maior em relação à observada para Minas Gerais neste mesmo ano $^{11}$. Estudos têm demonstrado que a gravidez na adolescência é mais comum entre aquelas de menor renda familiar e escolaridade. Geralmente, estas mulheres iniciam a vida sexual, engravidam e têm o parto mais cedo que as demais ${ }^{4,12}$. O Vale do Jequitinhonha, região onde se localiza o município desse estudo, é considerado uma das regiões mais pobres do Brasil. Se encontra localizado ao nordeste do estado de Minas Gerais e as cidades que fazem parte do Vale apresentam alguns dos piores indicadores socioeconômicos do país ${ }^{13}$. Esse fato pode, talvez, auxiliar na explicação da maior taxa de gravidez na adolescência em relação à que é observada no estado de Minas Gerais.

$\mathrm{O}$ percentual de todas as mães incluídas no presente estudo, que moravam sem o companheiro foi 2,4 vezes maior entre mães adolescentes em relação às mães adultas 59,7\% e 24,7\%, respectivamente. Viver sem o companheiro é um dos fatores que pode interferir na adesão ao pré-natal ${ }^{6}$. Pensar em uma assistência pré-natal de qualidade, que visa atender às necessidades que vão além das questões biológicas, perpassa pela inserção do companheiro nesse contexto. $\mathrm{O}$ acompanhante é reconhecido pelas políticas de saúde e pela própria gestante como componente importante na validação do atendimento com qualidade. Apesar de estar aumentando o número de consultas de pré-natal por gestante que realiza o acompanhamento na rede pública de saúde, é necessário ainda, dotá-la de maior qualidade, e uma das estratégias para que isso seja alcançado pode ser a inclusão do companheiro no contexto do pré-natal ${ }^{14}$.

Quanto ao uso de álcool e cigarro na gestação, pesquisa realizada com 433 puérperas de uma maternidade pública do Rio de Janeiro, verificou que $7,4 \%$ das puérperas relataram uso de álcool durante a gestação e 5,5\% relataram uso de cigarro $^{15}$, porcentagem inferior à encontrada na presente pesquisa. Ainda de acordo com esse estudo, as mulheres que viviam sem o companheiro tiveram chance quase três vezes maior de ingerir álcool e cigarro na gestação em comparação às que viviam com o companheiro ${ }^{15}$. Pesquisa realizada na Dinamarca, Islândia, Noruega e Suécia, evidenciou que mulheres que iniciam o tabagismo e uso de álcool precoce (antes dos 15 anos de idade) estão mais susceptíveis a experiências de gravidez na adolescência, comparado às mulheres que começaram a fumar ou beber mais tarde ${ }^{16}$. Com isso, evidencia-se a importância de profissionais da saúde direcionarem ações de prevenção ao uso dessas substâncias, voltadas aos adolescentes.

Estudo realizado com gestantes nos Estados Unidos descreve que a porcentagem do uso de cigarro foi $18,9 \%$ e o de álcool foi de $10 \%{ }^{17}$, divergente do encontrado nesse estudo, em que a porcentagem de uso de bebida alcóolica foi maior que a do uso de cigarro. O consumo de álcool durante a gestação é prejudicial, tanto para a mãe quanto para o feto, uma vez que o etanol atravessa facilmente a barreira placentária, podendo causar efeitos teratogênicos no feto. Os produtos derivados do cigarro também passam facilmente pela placenta. Além disso, a placenta de mães tabagistas apresenta características sugestivas de hipoperfusão, e, como consequência, há uma maior incidência de retardo do crescimento intrauterino, descolamento prematuro de placenta e rotura prematura das membranas ovulares ${ }^{18}$.

O pré-natal revela-se como sendo o momento ideal para intervenção e prevenção do uso de substâncias prejudiciais para mãe e filho, é preciso que haja uma relação de intensa confiança entre as equipes de saúde, a gestante e seus familiares, para que possam identificar os fatores de risco garantindo, assim, que melhores oportunidades de intervenção ocorram ${ }^{19}$.

Com relação aos problemas de saúde durante a gestação, a anemia, seguida da ITU foram as complicações gestacionais mais frequentes. Tais achados estão de acordo com o citado nos Manuais do Ministério da Saúde ${ }^{20,21}$, com os resultados encontrados na literatura nacional ${ }^{4,22} \mathrm{e}$ internacional ${ }^{23,24}$ e são similares ao encontrado nessa pesquisa. Durante a gestação, há aumento do volume plasmático em até $50 \%(1.000 \mathrm{ml})$ e do número total de hemácias circulantes em cerca $25 \%(300 \mathrm{ml})$, para suprir as necessidades 
do crescimento uterino e fetal. Por esse motivo, mesmo mulheres saudáveis, frequentemente apresentam queda fisiológica dos níveis de hematócrito e hemoglobina durante a gravidez. Além da anemia fisiológica da gravidez, é comum a presença de anemias secundárias a outras causas, principalmente aquelas devidas às carências de vitaminas e minerais ${ }^{20,21}$. A prevalência de anemia em gestantes de países desenvolvidos e em desenvolvimento é de cerca de $22,7 \%$ e $52,0 \%$, respectivamente ${ }^{4}$.

A ITU é, também, frequente na gestação, sua prevalência é estimada em $20 \%$. Em consonância ao encontrado nesse estudo, uma pesquisa realizada no estado do Rio Grande do Sul evidenciou que 2,9\% das mulheres da pesquisa haviam sido internadas para tratamento da infecção do trato urinário, evidenciando ainda que essa porcentagem foi maior em mulheres com menor nível econômico, mais jovens, com menor escolaridade e que viviam sem o companheiro ${ }^{22}$.

As síndromes hipertensivas são a primeira causa de morte materna no Brasil e determinam o maior número de óbitos perinatais. Duas formas de hipertensão podem complicar a gestação, que são a hipertensão preexistente (crônica - antes de 20 semanas de gestação) e a hipertensão induzida pela gestação (pré-eclampsia/eclâmpsia), podendo ocorrer isoladamente ou de forma associa$\mathrm{da}^{21,25}$. Pesquisa realizada no município do Rio de Janeiro encontrou 8,4\% de gestantes com hipertensão arterial. Entre as hipertensas predominaram aquelas com mais de 35 anos de idade, obesas e multíparas. A hipertensão arterial na gestação também está ligada à risco significativamente aumentado para parto pré-termo, recém-nascido com baixo peso ao nascer, desfechos perinatais desfavoráveis, além do aumento expressivo do número de neonatos com sequelas quando sobrevivem as lesões da hipóxia cerebral ${ }^{25,26}$.

Diante dos principais problemas de saúde durante a gestação, os profissionais das equipes de saúde devem estar capacitados para prestar uma adequada assistência de pré-natal em todos os níveis de atenção à saúde da mulher, de modo a identificar as possíveis situações de risco e o seu adequado manejo. É importante, para as unidades de saúde, adequar o número de atendimentos no pré-natal e grupos educativos, a fim de possibilitar maior tempo para esclarecimentos dos riscos e prevenção das doenças na gravidez.

$O$ início precoce da assistência pré-natal é recomendado pelo Ministério da Saúde e pelo Programa de Humanização no Pré-natal e Nascimento (PHPN), que recomenda a realização de, no mínimo, seis consultas de acompanhamento pré-natal, sendo, preferencialmente, uma no primeiro trimestre, duas no segundo trimestre e três no terceiro trimestre da gestação ${ }^{27,28}$. Em consonância com o Ministério da Saúde, neste estudo, a maioria das puérperas iniciou o pré-natal no período adequado e realizou mais de 6 consultas. Entretanto, observou-se que as adolescentes iniciam mais tardiamente e realizaram menos consultas se comparadas às adultas. Destaca-se que a adequação do número de consultas foi superior à adequação do início precoce, esse resultado provavelmente pode ser devido ao fato de uma maior realização de consultas no último trimestre gestacional.

Quanto ao local de realização do pré-natal, estudos também apontam que, a maioria das mulheres brasileiras realizam as consultas no serviço público de saúde. Sendo um pouco maior essa porcentagem entre as mulheres que residem nas regiões Norte e Nordeste, em mulheres de menor idade e escolaridade, sem companheiro e com maior número de gestações e desfechos negativos anteriores ${ }^{8,29}$. Tais informações corroboram as encontradas nessa pesquisa. Assim como nas consultas de pré-natal, a cobertura de parto da maioria das puérperas desse estudo também foi realizada no sistema público de saúde. Entre as adolescentes, esta proporção foi maior em relação às adultas. Uma possível explicação seria que, a gratuidade da rede pública leva àqueles de menor recurso a procurar por ela, o que inclui a quase totalidade dos partos entre as adolescentes ${ }^{8}$.

Quanto ao tipo de parto, a $\mathrm{OMS}^{9}$ considera que não há justificativa para um percentual superior a $15 \%$ de cesárea em nenhuma região do mundo. No Brasil, essa taxa varia entre 15\% a $45 \%$ do total de nascimentos ${ }^{19}$, sendo que em Minas Gerais a taxa de cesárea, em 2010, foi de $54,16 \%{ }^{30}$, muito distante daquilo que mundialmente é recomendado. Estudos têm evidenciado que há um aumento na taxa de cesarianas em todo Brasil e, similarmente ao encontrado nesse estudo, a idade materna tem sido diretamente relacionada à maior prevalência de cesarianas, em que mulheres de 30 anos ou mais têm maior probabilidade de realizarem partos cesarianos do que mulheres com idade inferior a $20 \operatorname{anos}^{7,22,31}$.

A cesariana, quando corretamente indicada, traz benefícios para a mãe e para o bebê, entretanto, potenciais desvantagens têm sido descritas em estudos, como: maior morbimortalidade materna, efeitos psicológicos adversos, problemas em futuras gestações (como ruptura da cicatriz uterina) e morbidade neonatal ${ }^{19,31,32}$. Evidencia- 
se a necessidade de políticas públicas voltadas à redução das taxas de cesariana; as gestantes devem ser corretamente avaliadas pelos profissionais da saúde e deve ser realizado cuidadoso monitoramento da condição fetal, sendo a cesárea uma indicação médica precisa.

\section{Considerações finais}

O caráter regional desse estudo pode ser considerado como uma limitação, uma vez que se trata de uma maternidade de menor porte, o que resultou em um menor número amostral. No entanto, o estudo alcançou seus objetivos, uma vez que demonstrou, com significância estatística, que as mães adolescentes estiveram em desvantagem em relação às demais mães, no que diz respeito tanto às características socioeconômicas quanto à assistência recebida no pré-natal. As adolescentes possuíam pior nível de renda familiar, viviam sem companheiro, realizaram um menor número de consultas de pré-natal e iniciaram estas consultas mais tardiamente em comparação com as adultas.

Os resultados evidenciaram a necessidade de ações voltadas para a saúde da mulher, em especial das adolescentes, no que diz respeito ao prénatal abrangendo planejamento familiar, saúde sexual e reprodutiva, consequências do uso de álcool, cigarro e outras drogas na gestação, principais doenças e suas complicações, entre outros assuntos pertinentes ao ciclo gravídico puerperal. Evitando assim, possíveis complicações que terão impactos não somente na vida das adolescentes, como também na mortalidade materno infantil e na saúde pública.

\section{Colaboradores}

LAV Santos participou da coleta, interpretação, análise dos dados e redação do artigo. RCR Lima e AF Rocha participaram da coleta, interpretação e análise dos dados. MO Lara, EM Rocha e GC Ribeiro participaram da elaboração do projeto, delineamento, análise e interpretação dos dados, revisão crítica e aprovação da versão a ser publicada. JCR Glória participou da revisão crítica e aprovação da versão a ser publicada. 


\section{Referêencias}

1. Cesar JA, Sassi RAM, Chica DAG, Mano PS, Goulart SMF. Características sociodemográficas e de assistência à gestação e ao parto no extremo sul do Brasil. Cad Saude Publica 2011; 27(5):985-994.

2. Santos NLAC, Costa MCO, Amaral MTR, Vieira GO, Bacelar EB, Almeida AHV. Gravidez na adolescência: análise de fatores de risco para baixo peso, prematuridade e cesariana. Cien Saude Colet 2014; 19(3):719-726.

3. Ferreira RA, Ferriani MGC, Mello DF, Carvalho IP, Cano MA, Oliveira LA. Análise espacial da vulnerabilidade social da gravidez na adolescência. Cad Saude Publica 2012; 28(2):313-323.

4. Barreto MS, Mathias TAF. Cuidado à gestante na atenção básica: relato de atividades em estágio curricular. Rev Rene 2013; 14(3):639-648.

5. Domingues RMSM, Hartz ZMA, Dias MAB, Leal MC Avaliação da adequação da assistência pré-natal na rede SUS do Município do Rio de Janeiro, Brasil. Cad Saude Publica 2012; 28(3):425-437.

6. Brasil. Ministério da Saúde (MS). Departamento de Informática do SUS. DATASUS. Indicadores de cobertura. Brasília: MS; 2010.

7. Sass A, Gravena AAF, Pelloso SM, Marcon SS. Resultados perinatais nos extremos da vida reprodutiva e fatores associados ao baixo peso ao nascer. Rev Gaúcha Enferm 2011; 32(2):352-358.

8. Fonseca SC, Monteiro DAS, Pereira CMSC, Scoralick ACD, Jorge MG, Rozario S. Desigualdades no pré-natal em cidade do sudeste do brasil. Cien Saude Colet 2014, 19(7):1991-1998

9. Organização Mundial da Saúde (OMS). Declaração da OMS sobre Taxas de Cesáreas. [acessado $2015 \mathrm{Jul}$ 17]. Disponível em: http://apps.who.int/iris/bitstream/10665/161442/3/WHO_RHR_15.02_por.pdf

10. Brasil. Ministério da Saúde (MS). Departamento de Informática do SUS (Datasus). Informações de Saúde. Brasília: MS; 2010.

11. Brasil. Ministério da Saúde (MS). Indicadores de fatores de risco e proteção. Brasília: MS; 2010.

12. Diniz E, Koller SH. Fatores Associados à Gravidez em Adolescentes Brasileiros de Baixa Renda. Paidéia 2012; 22(53):305-314.

13. Guedes DP, Mendes RR. Crescimento físico e estado nutricional de escolares do Vale do Jequitinhonha, Minas Gerais, Brasil. Rev Bras Cineantropom Desempenho Hum 2012; 14(4):363-376.

14. Cabrita BAC, Silveira ES, Souza AC, Alves VH. A ausência do companheiro nas consultas de pré-natal: desafios e conquistas. R. pesq.: cuid. fundam. online 2012; 4(3):2645-2654

15. Freire K, Padilha PC, Saunders C. Fatores associados ao uso de álcool e cigarro na gestação. Rev Bras Ginecol Obstet 2009; 31(7):335-341.

16. Hansen BT, Kjær SK, Munk C, Tryggvadottir L, Sparén P, Jenssen MH, Liaw KL, Nygård M. Early smoking initiation, sexual behavior and reproductive health $-\mathrm{a}$ large population-based study of Nordic women. Preventive Medicine 2010; 51(1):68-72.

17. Havens JR, Simmons LA, Shannon LM, Hansen WF Factors associated with substance use during pregnancy: results from a national sample. Drug Alcohol Depend 2009; 99(1-3):89-95.
18. Zhang L, Chica DAG, Cesar JA, Sassi RAM, Beskow B, Larentis N, Blosfeld T. Tabagismo materno durante a gestação e medidas antropométricas do recém-nascido: um estudo de base populacional no extremo sul do Brasil. Cad Saude Publica 2011; 27(9):1768-1776.

19. Brasil. Ministério da Saúde (MS). Atenção ao pré-natal de baixo risco. Brasília: Editora do Ministério da Saúde; 2013.

20. Brasil. Ministério da Saúde (MS). Manual técnico: Gestação de alto risco. $5^{\mathrm{a}}$ ed. Brasília: MS; 2010.

21. Brasil. Ministério da Saúde (MS). Manual Técnico: Prénatal e puerpério, atenção qualificada e humanizada. $2^{\mathrm{a}}$ ed. Brasília: MS; 2006.

22. AA, Albernaz EP. Prevalência e fatores associados à internação hospitalar para tratamento da infecção do trato urinário durante a gestação. Rev Bras Ginecol Obstet 2013; 35(5):199-204.

23. Quintero R, Muñoz MN, Álvarez LE, Medina GA. Estado nutricional y seguridade alimentaria en gestantes adolescentes. Pereira 2009. Invest Educ Enferm 2010; 28(2):204-213.

24. Hurtado AR, Ríos AG, Ortiz JAR. Validez del parcial de orina y el Gram en el diagnóstico de infección del tracto urinario en el embarazo. Hospital Simón Bolívar, Bogotá, Colombia, 2009-2010. Rev Colomb Obstet Ginecol 2013; 64(1):53-59.

25. Vettore MV, Dias M, Domingues RMSM, Vettores MV, Leal MC. Cuidados pré-natais e avaliação do manejo da hipertensão arterial em gestantes do SUS no município do Rio de Janeiro, Brasil. Cad Saude Publica 2011; 27(5):1021-1034.

26. Aggarwal AK, Jainb V, Kumara R. Validity of verbal autopsy for ascertaining the causes of stillbirth. Bull World Health Organ 2011; 89(1):31-40.

27. Brasil. Ministério da Saúde (MS). Portaria 1.459, de 24 de junho de 2011. Institui, no âmbito do Sistema Único de Saúde - SUS - a Rede Cegonha. Diário Oficial da União 2011; 25 jun.

28. Brasil. Ministério da Saúde (MS). Programa de Humanização no Pré-Natal e Nascimento. Brasília: MS; 2002.

29. Vasconcellos MTL, Silva PLN, Pereira APE, Schilithz AOC, Junior PRBS, Szwarcwald CL. Sampling design for the Birth in Brazil: National Survey into Labor and Birth. Cad Saude Publica 2014; 30(Supl.):S1-S10.

30. Brasil. Ministério da Saúde (MS). Departamento de Informática do SUS (Datasus). Indicadores de cobertura. Proporção de partos cesáreos. Brasília: MS; 2010.

31. Meller FO, Schäfer AA. Fatores associados ao tipo de parto em mulheres brasileiras: PNDS 2006. Cien Saude Colet 2011; 16(9):3829-3835.

32. Gama SGN, Viellas EF, Schilithz AOC, Filha MMT, Carvalho ML, Gomes KRO, Costa COM, Leal MC. Factors associated with caesarean section among primiparous adolescents in Brazil, 2011-2012. Cad Saude Publica 2014; 30(Supl.):S1-S11.

Artigo apresentado em 22/12/2015

Aprovado em 18/05/2016

Versão final apresentada em 20/05/2016 
